\title{
Grundlagen der Rechtsphänomenologie
}

\section{Eine kritische Darstellung der Rechtsphänomenologie von Adolf Reinach und Wilhelm Schapp zu den} apriorischen Grundlagen des Privatrechts

\author{
Von Kai P. Purnhagen, LL.M. (Wisconsin-Madison), M. Res. (Law), MJI
}

Die Rechtsphänomenologie ist eine Wesensforschung des Rechts. Adolf Reinach und Wilhelm Schapp haben sich dieser angenommen und jeweils ihr eigenes System der phänomenologischen Analyse des Privatrechts vorgestellt, die hier in Grundzügen dargestellt wird. Am Ende des Beitrags werden Stärken und Schwächen dieser Ansätze aufgezeigt.

Die Frage nach dem, was Recht ist, wie es zu definieren sei und woher es stammt, beschäftigt Juristen und Philosophen schon seit Jahrtausenden. Dabei ist die Fülle der Erklärungsmuster und Deutungsversuche kaum zählbar. Eine Darstellung, die auch nur annähernd den Anspruch auf Vollständigkeit erhebt, wäre sicherlich von Anfang an zum Scheitern verurteilt.

Diese Suche nach dem »Recht« als Gegenstand und seinen möglichen apriorischen Grundlagen ist nicht, wie man auf den ersten Blick vermuten könnte, eine reine Glasperlenspielerei im Elfenbeinturm eines bereits vergangenen Jahrhunderts. Sie hat vielmehr gerade heute hohe Praxisrelevanz. Dem Europäischen Gerichtshof beispielsweise genügt es, gem. Art.220 EG zur »Wahrung des Rechts" berufen zu sein, um bereits vorhandene ungeschriebene europäische Grundrechte zu finden und verweist dabei auf die »gemeinsamen Verfassungsüberlieferungen ${ }^{1}$. Mehr noch: In Zeiten, in denen nur aus den "gemeinsamen Prinzipien des Bürgerlichen Rechts $\ll^{2}$ ein kodifiziertes europäisches Privatrecht geschaffen werden soll, obwohl hierzu keine oder zumindest unzureichende Kompetenz auf Gemeinschaftsebene besteht ${ }^{3}$, müsste solche Forschung nach apriorischen Grundlagen des Bürgerlichen Rechts eigentlich mit Nachdruck verfolgt werden. Dennoch fristet sie gerade im Privatrecht ein Nischendasein und glänzt allenfalls durch ihre Abwesenheit. In diesem Beitrag soll daher ein Teilbereich dieser größtenteils in Vergessenheit geratenen Forschung dargestellt und näher auf die damit zusammenhängenden Probleme eingegangen werden.

\section{A. Ausgangspunkt der Phänomenologischen Rechts- forschung: Bruch mit Hergebrachten Theorien über das Wesen des Rechts}

Was ist Recht und was ist gerecht? Recht besteht aus Paragraphen, könnte man meinen. Warum gibt es dann aber solche Gesetze, die man als ungerecht empfindet, und warum sind

* Forscher am Europäischen Hochschulinstitut in Florenz und Lehrbeauftragter an der Universität Luzern. Ich bin Til Kappen zu großem Dank verpflichtet, der dieses Projekt mit großem Interesse und sehr kritisch begleitet hat. Fehler sind meine eigenen.

1 EuGH Rs. 11/70 Internationale Handelsgesellschaft ./. Einfuhr- und Vorratsstelle Getreide, Slg. 1970, 1125, 1134 f, Rdn. 2-4. Siehe hierzu auch Purnhagen/Rebasti/Bernhardt Reich der Richter? 1 European Journal of Legal Studies (No. 2) $3 \mathrm{f}$., abrufbar unter www.ejls.eu.

2 Die European Research Group on Existing EC Private Law und The Study Group on a European Civil Code gehen davon aus, dass hinter den EGRegulierungen allgemeingültige Prinzipien des Privatrechts stünden, die man als kodifiziertes europäisches Privatrecht darstellen könne. Dass diese Prinzipien teilweise sehr weitgehend und detailliert sein sollen, lassen die bereits veröffentlichten Werke dieser Gruppen erkennen, siehe beispielsweise http://www.sgecc.net/pages/en/texts/index.draft_articles.htm.

3 Siehe hierzu inter alia Weatherill European Private Law and the Constitutional Dimension in: Cafaggi (Hrsg.) The Institutional Framework of European Private Law 2006, 79-107; Micklitz An Expanded and Systemized Community Consumer Law as an Alternative or Complement? 13 European Business Law Review 583 (584). 
andererseits als ungerecht empfundene Lebenssachverhalte nicht rechtlich sanktioniert? Hier soll sich diesen Fragen aus einer für den Juristen ungewöhnlichen Perspektive genähert werden, aus der Perspektive der Rechtsphänomenologie. Diese Wissenschaft geht auf Edmund Husserl zurück und wurde vor allem durch den Rechtsphilosophen Adolf Reinach ausdifferenziert. Wilhelm Schapp, ebenfalls ein Schüler Husserls, führte zunächst das Werk Reinachs kritisch fort, entwickelte später jedoch eine eigene Geschichtenphänomenologie, die sich von Reinach abwandte. Sie alle versuchten, auf Grundlage der Phänomenologie eine Antwort darauf zu finden, was Recht ist. Oder mit den Worten der Phänomenologie: Was das Wesen des Rechts ist. Die hier dargestellten Theorien sind aufgrund des vorhandenen Materials auf Einzelaspekte des Bürgerlichen Recht beschränkt, auch wenn Reinach und Schapp ihre Theorien auch als Methode, die über das Bürgerliche Recht hinaus angewendet werden kann, verstanden haben ${ }^{4}$.

Auch wenn es schwierig ist, eine gemeinsame Linie aus den vielfachen Erklärungsmustern zur Frage nach dem Dasein des Rechts zu destillieren ${ }^{5}$, so kann man rückschauend doch mit einiger Gewissheit sagen, dass die meisten Denkschulen in Europa davon ausgehen, dass Recht grundsätzlich »in seiner Entstehung an die Existenz des Staates " gebunden ist ${ }^{6}$. Manche gehen sogar weiter und sehen als Recht nur an, "was in einem bestimmten Verfahren von einer hierfür eingerichteten staatlichen Einrichtung oder einer vom Staat dazu ermächtigten Stelle für Recht erklärt worden ist $\aleph^{7}$. Als einziger anerkannter Bruch mit diesem Grundsatz gilt die Anerkennung der Existenz gewisser Naturrechte, die jedem Menschen unabhängig von seiner Stellung in Staat, Gesellschaft, Familie, Beruf, Religion und Kultur bereits dadurch zustünden, dass er als Mensch geboren ist ${ }^{8}$. Jedoch sei dieser Bruch gewissermaßen dadurch geheilt, dass die Staaten (zumindest Europas) diese Menschenrechte anerkannt und positiviert hätten (vgl. Art. 1 II GG, EMRK) ${ }^{9}$. Die Frage, ob ein von dem Staat unabhängiges Recht existiert, könne aus diesem Grund dahingestellt bleiben ${ }^{10}$. Es bleibt nach der vorherrschenden Ansicht somit dabei, dass das Recht in seiner Entstehung an die Existenz des Staates gebunden ist. In der Jurisprudenz scheint daher große Einigkeit in dem Punkt zu bestehen, dass das Recht nicht losgelöst von der rechtsetzenden Gewalt, dem Staat, beurteilt werden kann. Recht könnte man daher so verstehen, dass es "nichts anderes als die in der staatlichen Gemeinschaft herrschende Ordnung « ${ }^{11}$ ist. Gegen dieses Postulat wandten sich Reinach und Schapp.

\section{B. Die Rechtsphänomenologie von Adolf Reinach und Wilhelm Schapp}

Reinach stellt dieser Ansicht seine »fundamental andere« rechtsphänomenologische Untersuchung von den apriorischen Grundlagen des Bürgerlichen Rechts entgegen ${ }^{12}$.

Es gebe an sich seiende rechtliche Gebilde, die das Recht vorfände und gerade nicht erzeuge ${ }^{13}$.

Auf diesem Gedankengang aufbauend führt Wilhelm Schapp seine eigenen Überlegungen in seinen Frühwerken fort ${ }^{14}$. Auch Wilhelm Schapp geht zunächst von gewissen Vorgegebenheiten aus, die Grundlage eines jeden positiven Rechtes seien ${ }^{15}$. Diese Vorgegebenheiten würden nicht aus dem positiven Recht gewonnen, das positive Recht fände sie vielmehr vor und müsse nur noch aus ihnen auswählen ${ }^{16}$. An dieser Stelle mag der Hinweis auf die gemeinsame Grundlage beider Arbeiten genügen. Die spätere Geschichtenphänomenologie Wilhelm Schapps wird sodann gesondert und nur überblicksartig behandelt, da sie sich nicht ohne weiteres in den Kontext der wesentlich früher erschienenen Werke von Wilhelm Schapp und Reinach einfügen lassen.

\section{I. Überblick über die Methode der klassischen Phänomenologie}

Um die Rechtsphänomenologie der Autoren erfassen zu können, erscheint es zweckmäßig, sich zunächst einen Überblick über die Methode der klassischen Phänomenologie zu verschaffen. Dieser Überblick hat nicht den Anspruch, vollständig und abschließend zu sein. Er soll lediglich einen Einblick in die Methode der Phänomenologie geben, deren Kenntnis unerlässlich für das Verständnis der Rechtsphänomenologie von Reinach und Wilhelm Schapp ist. Ausgangspunkt jeder phänomenologischen Überlegung ist das Verhältnis vom Satz zum Sachverhalt ${ }^{17}$.

\section{Satz, Begriff und Gegenstand}

Der Satz ist das Grundgebilde der Phänomenologie ${ }^{18}$. Um diesem Satz näher zu kommen, untersucht die klassische Phänomenologie die Begriffe, aus denen er besteht ${ }^{19}$. Beispielsweise enthält der Satz »Der Baum ist rot« die Begriffe »Baum« und $»$ Der $«$.

\section{a) Die Selbstgegebenheit der Gegenstände}

Den Begriffen können Gegenstände entsprechen ${ }^{20}$. Den Gegenstand »Baum « erfassen wir wiederum durch unsere Wahrnehmung. Daher kann man von einer Selbstgegebenheit der Gegenstände sprechen ${ }^{21}$. An dieser Stelle bleibt somit festzuhalten, dass der Phänomenologe von einer Selbstgegebenheit der wahrnehmbaren Gegenstände ausgeht, auf denen seine Überlegungen aufbauen. In unserem Beispiel entspricht der Begriff »Baum» dem Gegenstand »Baum».

\section{b) Das »Wesen « der Gegenstände}

Diese Begriffe können nun weiter untersucht werden nach einem »Wesen« des Begriffes, einem ihm »eigenartigen Sein «22. So würde man im Beispiel davon ausgehen, dass das Wesen eines Bau-

4 Siehe nur Reinach Die apriorischen Grundlagen des bürgerlichen Rechts 690 Fn. 1 in: Husserl, Jahrbuch der Philosophie und phänomenologischen Forschung, Bd. 1, 1922, 683-847.

5 Eine weitgehend gelungene Kurzdarstellung der verschiedenen Ansichten findet sich bei Arzt Einführung in die Rechtswissenschaft 2. Aufl. 1996, $19-23$.

6 Avenarius Die Rechtsordnung der Bundesrepublik Deutschland 3. Aufl. 2002, 1.113. Ob dies tatsächlich der "rule of recognition«, die H. L. A. Hart einst formulierte, gerecht werden würde und ob eine solche »rule of recognition« überhaupt notwendig ist, soll hier offen bleiben.

7 Ebenda.

8 Oestreich Die Idee der Menschenrechte 3. Aufl. 1969, 9.

9 Arzt Einführung in die Rechtswissenschaft 20 unten.

10 Avenarius Die Rechtsordnung der Bundesrepublik Deutschland 1.122, der eine Existenz von Naturrechten dahingestellt sein lässt, da sie ohnehin durch das Grundgesetzt gewährleistet werden.

11 Zitat wird Aristoteles zugeschrieben, so auch Brox/WALKer Allgemeiner Teil des BGB 32. Aufl. 2008 Rdn. 1, der jedoch die Ordnung nur dann als Recht begreift, wenn sie an der Idee der Gerechtigkeit ausgerichtet ist.

12 Reinach Die apriorischen Grundlagen des bürgerlichen Rechts 688 in Husserl, Jahrbuch der Philosophie und phänomenologische Forschung, Bd. 1 Halle 1922, 683-847.

13 Ebenda.

14 Wilhelm Schapp Die neue Wissenschaft vom Recht I. Band »Der Vertrag als Vorgegebenheit « (Im Folgenden nur noch »Der Vertrag als Vorgegebenheit «) 1930, 182. Vgl. auch JAN SCHA P P Sein und Ort der Rechtsgebilde, eine Untersuchung über Eigentum und Vertrag 1968, 25.

15 Wilhelm Schapp Der Vertrag als Vorgegebenheit 5; 35 f.; 49; vgl. hierzu auch Jan Schapp Sein und Ort der Rechtsgebilde $38 \mathrm{f}$.

16 Wilhelm Schapp Der Vertrag als Vorgegebenheit $35 \mathrm{f}$.

17 Wilhelm Schapp In Geschichten verstrickt, Zum Sein von Mensch und Ding 3. Aufl. 1985 S. 169.

18 Ebenda 173

19 Ebenda 169

20 Ebenda 169

21 Ebenda 170

22 Ebenda 171 
mes ist, einen Stamm zu haben. Nun würde der Begriff des "Stamms" untersucht werden usw. Diese Begriffe fügen sich zur Einheit des Satzes zusammen ${ }^{23}$.

\section{Satz und Sachverhalt}

Wenn nun behauptet wird, dass nicht die Begriffe, die im Sinne der Phänomenologie das Primäre sind, sondern der Satz das Grundgebilde der Phänomenologie ist, so ist dies erklärungsbedürftig. Sätze können gedacht werden ${ }^{24}$. Wird der Satz gesagt, so hat der Sprechende jedoch nicht den Satz an sich, sondern den Sachverhalt vor Augen ${ }^{25}$. Der Sprechende sagt den Satz, versucht jedoch, ihn sich als Sachverhalt vorzustellen ${ }^{26}$. Vielleicht wird dieses System dadurch klarer, wenn man dabei davon ausgeht, dass der Sprechende immer an seine subjektiven Empfindungen beim Denken des an sich objektiv eindeutigen Satzes ${ }^{27}$ gebunden ist, woraus sich der Sachverhalt ergibt. Zwischen Satz und Sachverhalt lässt sich eine Beziehung herstellen, die als wahr oder falsch zu kennzeichnen ist ${ }^{28}$. Um bei unserem Beispiel zu bleiben: Der Sprechende sieht einen roten Laternenmast nur flüchtig und glaubt, er sei ein Baum. Somit sagt er den Satz »Der Baum ist rot « und hat damit den Sachverhalt eines roten Baumes vor Augen. Dieser Satz ist jedoch nicht wahr, weil er mit dem gemeinten Sachverhalt nicht übereinstimmt.

Um diese Wahrheit und Falschheit festzustellen, beziehen wir uns auf der Sachverhaltsebene wiederum auf die Gegenstände, deren Wesen wir bei den "Begriffen« schon untersucht haben. Ein Rückgriff auf die Gegenstände ist aus der Sicht eines Phänomenologen legitim, da sie durch ihre Wahrnehmbarkeit selbstgegeben sind. Somit kann man mit einiger Sicherheit davon ausgehen, dass der gedachte Satz falsch ist, weil das "Wesen des Baumes ist, einen Stamm zu haben. Der Sachverhalt jedoch enthielt einen Laternenmast, der keinen Stamm hat.

\section{Zusammenfassung}

Der Phänomenologe denkt sich also einen Satz, indem er sich einen Sachverhalt vorstellt, während er einen Gegenstand wahrnimmt. Dieser Satz kann dadurch verifiziert werden, dass der gemeinte Sachverhalt existiert, wobei hier wiederum auf die selbstgegebenen Gegenstände Bezug genommen wird. Lapidar formuliert kann man wohl sagen, dass der Phänomenologe zunächst die Dinge hinnimmt, wie sie der Einzelne wahrnimmt. Ob diese Wahrnehmung richtig oder falsch ist, wird durch einen Vergleich der Wesenszüge der wahrgenommenen Sache (des gedachten Sachverhalts) mit den Wesenszügen, die diese Sache üblicherweise hat, ermittelt.

Dieser nur abrissartige Überblick über die klassische Phänomenologie mag genügen, um einen Einstieg in die Denkweise Reinachs und damit die Grundlegung für die Überlegungen von Wilhelm Schapp zu setzen ${ }^{29}$.

\section{II. Über die apriorischen Grundlagen bei Reinach}

Wenden wir uns mit diesen Überlegungen im Hinterkopf der Lehre Reinachs zu. »Von den rechtlichen Gebilden gelten apriorische Sätze $\ll^{30}$. Dieser Satz ist zugleich Ergebnis und Ausgangspunkt der Überlegungen Reinachs. Er bedient sich zur Verifikation dieser These den eben geschilderten Methoden der klassischen Phänomenologie ${ }^{31}$.

\section{Die rechtlichen Gebilde}

Somit stellt sich zunächst die Frage, was Reinach unter solchen »rechtlichen Gebilden" versteht. Gebilde entsprechen aus phänomenologischer Sicht zunächst den Gegenständen, die allerdings im Unterschied zu ihnen zeitlos sind (Beispielsweise Zahlen). Jedoch unterschieden sich die "rechtlichen Gebilde" von den üblichen Gebilden der klassischen Phänomenologie. Rechtliche Gebilde seien »Gebilde, die man allgemein als spezifisch rechtliche bezeichnet $^{32}$, wie Anspruch, Verbindlichkeit und Eigentum $^{33}$. Nun können Anspruch, Verbindlichkeit und Eigentum jedoch unter anderem erlöschen. Somit ist Wesensmerkmal dieser Gebilde gerade ihre Zeitlichkeit ${ }^{34}$. "Rechtliche Gebilde" seien daher Gegenstände einer ganz neuen $\mathrm{Art}^{35}$, die »nicht zur Natur im eigentlichen Sinne gehören, die weder physisch noch psychisch sind und die sich zugleich auch von allen ideellen Gegenständen durch ihre Zeitlichkeit unterscheiden ${ }^{36}$. Reinach bezeichnet die »rechtlichen Gebilde« auch als »rechtliche Begriffe $\ll^{37}$, was für den Juristen anschaulicher sein mag.

Eine genaue Definition der rechtlichen Gebilde gibt Reinach jedoch nicht. Folgt man der Argumentation von Karl Schumann und Billy Smith, sind die »rechtlichen Gebilde « bei Reinach weitgehend übereinstimmend mit den »Tatbeständen« in Ernst Belings $»$ Die Lehre vom Verbrechen $\aleph^{38}$. Beling definiert sehr wohl, was er mit einem Tatbestand meint: Ein Tatbestand ist der Umriss eines Verbrechenstypus ${ }^{39}$. In der Tat scheint Belings »Tatbestand « gewisse Gemeinsamkeiten mit den »Gebilden" Reinachs zu haben, da auch Beling den Begriff »Tatbestand « als raum- und existenzlos definiert ${ }^{40}$. Jedoch ist der »Tatbestand « in der Lehre Belings abhängig von dem durch das positive Recht bestimmten Verbrechenstypus ${ }^{41}$. Somit sind die »Tatbestände « gerade nicht durch Vorgegebenheiten beeinflusst, sondern durch dispositives Recht. Daher kann der Ansicht Smiths und Schumanns nicht gefolgt werden.

Diese rechtlichen Gebilde könne man erforschen wie »Zahlen und geometrische Gebilde $\ll^{42}$, und apriorische Sätze von ihnen bilden, die »streng formulierbar und evident einsichtig sind. $\aleph^{43}$ Als Schüler Husserls ${ }^{44}$ meinte Reinach damit jedoch nicht die empirische Untersuchung ${ }^{45}$. Vielmehr bezog er sich hierbei auf

23 Ebenda 169.

24 Ebenda.

25 Ebenda, jedoch für den Denkenden.

26 Ebenda 169.

27 Objektiv eindeutig ist der Satz deshalb, weil er aus selbstgegebenen Gegenständlichkeiten besteht.

28 Wilhelm Schapp In Geschichten verstrickt 170

29 Wilhelm Schapp hat seine rechtsphänomenologischen Untersuchungen an diejenigen Reinachs angeknüpft (Wilhelm Schapp Der Vertrag als Vorgegebenheit 182); vgl. auch Jan Schapp Sein und Ort der Rechtsgebilde 25 .

30 Reinach Die apriorischen Grundlagen des bürgerlichen Rechts, S. 689. 31 Ebenda.

32 Ebenda 688.

33 Ebenda, auch das Straf-, Staats- und Verwaltungsrecht habe solche Begriffe, die zu untersuchen wären. Reinach bezieht sich jedoch nur auf Begriffe des bürgerlichen Rechts (Ebenda 690 Fn. 1).

34 Ebenda 694.

35 Ebenda.

36 Ebenda $690 \mathrm{f}$

37 Ebenda 688.

38 Schummann/Smith Adolf Reinach: An intellectual Biography 13 abrufbar unter http://ontology.buffalo.edu/smith/articles/reinach_biography.pdf.

39 BeLing Die Lehre vom Verbrechen 1906, 110

40 Ebenda 111.

41 Ebenda $21 \mathrm{f}$.

42 ReINACH Die apriorischen Grundlagen des bürgerlichen Rechts 689.

43 Ebenda; dem wird entgegengehalten, dass das Recht grundsätzlich mit der phänomenologischen Methode nicht untersucht werden könne, da die durch die Wesenslehre der Phänomenologie vorgegebene "phänomenologische Reduktion« das Recht von vornherein ausschalten würde (IsAY Rechtsnorm und Entscheidung 1929, 46 in Bezug auf HusserL Erstes Buch: Allgemeine Einführung in die reine Phänomenologie 1950 $\$ 56)$. Dem hält Jan Schapp Sein und Ort der Rechtsgebilde 26 entgegen, dass das Recht lediglich als Wirklichkeit der natürlichen Welt ausgeschaltet und dadurch gerade die phänomenologische Methode vorgegeben werden würde.

44 Schummann/Smith Adolf Reinach: An intellectual Biography 8; GöDICKE Bereicherungsrecht und Dogmatik 2002, 63 (Fn. 66).

45 Das Selbstverständnis der Phänomenologie als Gegensatz zum Empirismus wird besonders deutlich bei der Formulierung Wilhelm Schapps, als 
die Methode der eben dargestellten klassischen Phänomenologie. Denken wir uns hierhin zurück, so können wir an die Stelle der Gegenstände die rechtlichen Gebilde im Sinne Reinachs setzen $^{46}$.

\section{a) Die Selbstgegebenheit der rechtlichen Gebilde}

Diese Gegenstände sind in der klassischen Phänomenologie durch die Möglichkeit der Wahrnehmung selbstgegeben ${ }^{47}$. Rechtliche Gebilde können jedoch nicht in diesem Sinne wahrgenommen werden, da sie gerade nicht physischer Natur sind ${ }^{48}$. Somit könnte man an ihrer Selbstgegebenheit zweifeln. Gerade diese Selbstgegebenheit der Gebilde ist jedoch für die klassische phänomenologische Methode von evidenter Wichtigkeit, da die Wahrheit des Verhältnisses von Satz und Sachverhalt durch den Rückblick auf die selbstgegebenen Gebilde und ihre Wesensgesetze erfolgt. Reinach behilft sich dadurch, dass er davon ausgeht, dass es ein Bewusstsein, ein "schlichtes Wissen" von den rechtlichen Gebilden gibt, vergleichbar mit dem von Zahlen ${ }^{49}$. Ein schlichtes Wissen von einem Gegenstand reiche jedoch auch bei einem Gegenstand nicht aus, um ihn als selbstgegeben hinzunehmen. Er müsse vielmehr wahrgenommen, beispielsweise gefühlt oder gesehen werden. Auch dies billigt Reinach in gewisser Art und Weise den rechtlichen Gebilden zu. Es gebe ein Erlebnis, das den rechtlichen Gebilden vorausgeht ${ }^{50}$. Als Beispiel führt er ein »Sich-Verpflichtet-Fühlen« bei einer eigenen Verbindlichkeit $\mathrm{an}^{51}$. Somit sind die rechtlichen Gebilde nach Ansicht Reinachs durch das bloße Wissen von ihnen und das Erlebnis der vorausgehenden Akte, dem Grund der Rechtgebilde, selbstgegeben ${ }^{52}$.

Hier bleibt festzuhalten, dass es Reinach offenbar nicht vermag, die Selbstgegebenheit der rechtlichen Gebilde aus sich heraus zu begründen, da sie eben nicht wahrnehmbar sind. Er bedient sich vielmehr der den Gebilden vorausgehenden Akten, um die Selbstgegebenheit der Gebilde zu erklären.

\section{b) Die Entstehung durch die sozialen Akte}

Somit kann die Rechtsphänomenologie Reinachs in vollem Umfang nur erfasst werden, wenn man schon bei den Überlegungen über die Selbstgegebenheit der rechtlichen Gebilde die sozialen Akte mit einbezieht ${ }^{53}$. Nach Meinung Reinachs entstehen die Rechtsgebilde erst durch den Vollzug von sozialen Akten ${ }^{54}$. Akte sind beispielsweise das Sich-Entschließen, das Verzeihen, das Loben, das Tadeln, das Einräumen, das Versprechen und das Verzichten ${ }^{55}$. Diese Akte sind jedoch nicht allesamt "soziale« Akte. Ein Akt ist nur als sozial anzusehen und damit von Bedeutung für die rechtlichen Gebilde, wenn er vernehmungsbedürftig ist, da er sonst nicht als Erlebnis vernommen werden könne ${ }^{56}$. Wie das Zusammenwirken von sozialen Akten und Rechtsgebilde nach den Vorstellungen Reinachs funktioniert, soll am Beispiel von Anspruch und Verbindlichkeit als Rechtsgebilde und das Versprechen und die Annahme als sozialem Akt erläutert werden ${ }^{57}$ :

»Kein Anspruch und keine Verbindlichkeit beginnt ohne "Grund« zu existieren oder erlischt ohne einen solchen Grund. ${ }^{58}$ Teilweise wird das Verhältnis von Rechtsgebilde und sozialen Akten in Reinachs Rechtsphänomenologie als UrsacheWirkung- Beziehung bezeichnet ${ }^{59}$.

\section{aa) Das Versprechen}

Somit wird zunächst ein Versprechen geäußert ${ }^{60}$. Dieses Versprechen ist von anderen, ähnlichen sozialen Akten wie dem Befehl ${ }^{61}$ scharf zu trennen. Subjektiv kann diese Trennung erfolgen über das »innere Erlebnis« des Befehlenden. So setzt der Befehl als »inneres Erlebnis $«^{62}$ des Befehlenden voraus, dass der Adressat das Befohlene ausführt ${ }^{63}$. Den Willen, dass etwas geschehe, hat auch der Versprechende. Nur soll der Inhalt des Versprechens nicht vom Adressaten, sondern vom Versprechenden ausgeführt werden $^{64}$. Objektiv lässt sich diese Trennung der sozialen Akte nur durch die äußere Erscheinung unterscheiden, durch »die Art des Sprechens, in Betonung, Schärfe und ähnlichen schwer fixierbaren Faktoren ${ }^{65}{ }^{65}$ lässt sich feststellen, ob ein Versprechen oder ein Befehl gegeben wurde.

Dies lässt sich wohl am Beispiel des Spazierengehens erläutern: A sagt zu B: »Gehe mit mir spazieren! « Damit intendiert A, dass B mit ihm widerstandslos spazieren geht. Sagt A hingegen zu B: »Ich gehe mit Dir spazieren!», so verspricht er dem B, mit ihm spazieren $\mathrm{zu}$ gehen. Zu klären ist jedoch, ob A durch dieses Versprechen nun schon eine Verpflichtung eingegangen ist und $B$ einen Anspruch hat oder ob es erst der Annahme des B bedarf ${ }^{66}$.

\section{bb) Anspruch und Verbindlichkeit}

Wie bereits dargestellt wurde, können rechtliche Gebilde nur durch das Erlebnis der vorausgehenden sozialen Akte entstehen. Daher ist es einsichtig, dass ein Anspruch auf Seiten des Versprechensadressaten erst entstehen kann, wenn dieser das Versprechen vernommen hat ${ }^{67}$. Aus diesem Grund ist es nicht erforderlich, dass zur Entstehung eines Anspruchs der Verspre-

Schüler Husserls habe man »(...)den Kampf mit den mächtigsten Richtungen in der Wissenschaft der Welt, mit der kantischen Philosophie und dem Empirismus (...) «aufgenommen. (Wilhelm SCHAPP Erinnerungen an Edmund Husserl 1976, 18).

46 Jan Schapp Sein und Ort der Rechtsgebilde 27, der deutlich macht, dass man sich in den Augen Reinachs in das Wesen dieser Gebilde »ebenso vertiefen « könne wie in das Wesen von anderen Gebilden.

47 Wilhelm Schapp In Geschichten verstrickt 170.

48 Reinach Die apriorischen Grundlagen des bürgerlichen Rechts 702.

49 Ebenda $695 \mathrm{f}$.

50 Ebenda 696.

51 Ebenda.

52 Ebenda 702.

53 Vgl. Jan Schapp Sein und Ort der Rechtsgebilde 27.

54 Reinach Die apriorischen Grundlagen des bürgerlichen Rechts 694 f; 702.

55 Reinach Die apriorischen Grundlagen des bürgerlichen Rechts $706 \mathrm{ff}$.; Diese Übersicht ist beispielhaft und nicht enumerativ. Für eine umfangreichere, ebenfalls nicht abschließende Aufzählung von Akten vgl. JAN Schap P Sein und Ort der Rechtsgebilde 27.

56 ReINACH Die apriorischen Grundlagen des bürgerlichen Rechts 707, Reinach spricht anschaulich davon, dass sich diese Akte in die Seele des Empfängers »einbacken" solle.

57 Nicht in die Arbeit einbezogen werden die Überlegungen Reinachs zu dem absoluten Rechtsgebilden (wie etwa dem Eigentum), da die Darstellung wesentlich komplizierter ist und somit den Anspruch der Arbeit, einen gut eingänglichen Überblick und einen ersten Zugang zu der Materie zu gewähren, nicht gerecht werden würde.

58 ReinaCH Die apriorischen Grundlagen des bürgerlichen Rechts 701.

59 Jan Schapp Sein und Ort der Rechtsgebilde 28, wohl auch Reinach selbst in Die apriorischen Grundlagen des bürgerlichen Rechts 693.

$60 \mathrm{Im}$ folgenden gehen wir nur von dem »Echtversprechen « aus, das sich vom Scheinversprechen, beispielsweise einer Scherzerklärung, unterscheidet (vgl. REınACH Die apriorischen Grundlagen des bürgerlichen Rechts $717 \mathrm{f}$.)

61 Reinach spricht von einer Ähnlichkeit des Versprechens und des Befehls (REINACH Die apriorischen Grundlagen des bürgerlichen Rechts 714).

62 Im Folgenden wird anstatt des »inneren Erlebnisses« der besseren Verständlichkeit halber vom »Willen « gesprochen

63 Reinach Die apriorischen Grundlagen des bürgerlichen Rechts 710 .

64 ReINACH Die apriorischen Grundlagen des bürgerlichen Rechts $714 \mathrm{f}$.

65 Reinach Die apriorischen Grundlagen des bürgerlichen Rechts 709.

66 Unterstellt, es wollten A und B einen Vertrag über das Spazierengehen schließen (den man normalerweise nach den Grundsätzen des Versprechens des täglichen Lebens mangels Rechtsbindungswillen verneinen würde), so käme dieser und mit ihm Anspruch und Verbindlichkeit nach den Vorschriften des BGB gem. $\$ 151$ S. 1 HS. 1 erst mit der Annahme des Antrags zustande. Somit müsste B erst das Angebot des A annehmen. Jedoch ist sich immer wieder vor Augen zu halten, dass Reinach gerade diese positivrechtliche Sphäre von der Wesenssphäre unterscheidet und somit beide getrennt zu betrachten sind (vgl. hierzu auch JAN SCHAPP Sein und Ort der Rechtsgebilde 29).

67 Reinach spricht von einem Innewerden, REINACH Die apriorischen Grundlagen des bürgerlichen Rechts $721 \mathrm{f}$. 
chensadressat einen weiteren sozialen Akt der Annahme vollzieht.

Ein Anspruch kann daher nicht entstehen, wenn der B das Versprechen des A, mit ihm spazieren zu gehen, überhaupt nicht wahrgenommen hat, weil er in einem anderen Raum gewesen ist. Hört er jedoch die Ausführung des A und verinnerlicht diese, so ist ihm ein Anspruch entstanden.

Reinach wendet diese Wesensgesetzlichkeiten nun auch für die Entstehung der Verbindlichkeit an. Eine Verbindlichkeit auf Seiten des Versprechenden könne nur entstehen, wenn der Adressat das Versprechen vernommen habe ${ }^{68}$. Hier hat nun Reinach nicht in der notwendigen Sorgfalt, die das von ihm entwickelte System verlangt, gearbeitet ${ }^{69}$. Wie bereits dargestellt wurde, ist der Anknüpfungspunkt für die Entstehung eines rechtlichen Gebildes das Erlebnis des ihm vorausgehenden sozialen Aktes bei demjenigen, den das Gebilde betrifft. Der Versprechende hat das Versprechen bereits verinnerlicht, bevor es den Adressaten erreicht. Er fühlt sich verpflichtet. Somit müsste eine Verbindlichkeit des Versprechenden in dem System Reinachs bereits mit der Verinnerlichung des sozialen Aktes durch den Versprechenden entstehen $^{70}$. Jedoch behilft sich Reinach damit, dass er von einer »eigenartigen Korrelativität « von Anspruch und Verbindlichkeit ausgeht, die darin bestehe, dass beide wechselseitig ineinander verflochten seien ${ }^{71}$. Aus diesem Grund könne eine Verbindlichkeit nicht ohne einen Anspruch entstehen ${ }^{72}$. Streng genommen ist dies jedoch kein Ergebnis, das in das Reinachsche System passt. Mithin müsste nach der von Reinach selbst angewandten Logik eine Verbindlichkeit schon mit dem Innewerden des Versprechens beim Versprechenden entstehen. Hiervon grundsätzlich zu unterscheiden sind die absoluten Verbindlichkeiten, die nicht durch Versprechungen entstehen ${ }^{73}$, sondern durch den Akt der Bestimmung durch den Gesetzgeber ${ }^{74}$.

Somit entsteht im Sinne Reinachs ein rechtliches Gebilde durch den Vollzug der sozialen Akte ${ }^{75}$. Beispielsweise entsteht ein Anspruch, wenn der Adressat eines Versprechens den Versprechensinhalt verinnerlicht hat.

\section{c) Zwischenergebnis}

An dieser Stelle bleibt zunächst festzuhalten, dass Reinach allergrößte Sorgfalt darauf verwendet, die Selbstgegebenheit der rechtlichen Gebilde zu erläutern. Er schafft ein System von sozialen Akten und deren Beziehungen zu den Gebilden, vermöge dessen diese zur Entstehung gelangen. Reinach kompensiert in gewisser Weise die fehlende Erkenntnismöglichkeit der rechtlichen Gebilde durch dieses komplexe System. Somit wurde zunächst umrissen, was Reinach unter den "rechtlichen Gebilden versteht. Nunmehr ist der zweite Teil der zentralen Aussage Reinachs zu untersuchen: die apriorischen Sätze.

\section{Die apriorischen Sätze}

Im Gegensatz zu den rechtlichen Gebilden, die Reinach eher beschreibt als sie zu definieren, umreißt er die apriorischen Sätze offenbar klar: »Jeder Sachverhalt, der im angegebenen Sinn allgemein ist und notwendig besteht, ist apriorisch ${ }^{76}$. So einleuchtend diese Aussage auf den ersten Blick sein mag, so schwierig ist sie jedoch zu handhaben. Was meint Reinach nun in seinem System mit einem Sachverhalt? Als Sachverhalt kann nun in unserem Beispiel allein die Tatsache gelten, dass A den B zum Spazierengehen auffordert. Wie nun diese Aufforderung ausgestaltet ist, ob sie befohlen wird oder ob A sie verspricht, kann nun mit dem Wesen des Rechtsgebildes verglichen und daran herausgearbeitet werden, ob ein derart vorgestelltes Rechtsgebilde vorliegt. Somit führt ein Vergleich des Sachverhalts mit den Wesensgesetzen der Rechtsgebilde dazu, den vorgestellten Sachverhalt zu verifizieren. Die Wesensgesetze der Rechtsgebilde sind daher das Vorgefundene ${ }^{77}$. Wie bereits dargestellt wurde, ist daher ein apriorischer Satz des (relativen)
Anspruchs beispielsweise, dass er aus einem Versprechen entspringt $^{78}$.

\section{Ergebnis}

Reinach hat mit seiner Rechtsphänomenologie somit die Grundlage für eine phänomenologische Erforschung des Rechts gelegt. Aus seiner Sicht lässt sich mit der klassischen phänomenologischen Methode ein Verhältnis von Sachverhalt und Rechtsgebilde herstellen, vermöge dessen überprüft werden kann, ob ein Rechtsgebilde entstanden ist oder nicht. Der objektive, allwissende Betrachter nimmt den Vollzug eines sozialen Aktes wahr, denkt sich ein Rechtsgebilde als Sachverhalt und vergleicht diesen Sachverhalt mit den Wesensgesetzen (den apriorischen Grundlagen) des Rechtsgebildes. Durch diesen Vergleich kann er bestimmen, ob es sich bei dem Vollzug der sozialen Akte um ein Rechtsgebilde handelt oder nicht.

\section{Kritik}

Reinachs Rechtsphänomenologie ist auf zahlreiche, in großen Teilen ablehnende Kritik gestoßen ${ }^{79}$. Dennoch darf die Leistung Reinachs, die insbesondere in der Herausarbeitung der Akte und der Rechtsgebilde begründet liegt, nicht unterschätzt werden. Seine Ausarbeitung der inneren und der äußeren Seite der sozialen $\mathrm{Akte}^{80}$ findet sich heute, fast ein Jahrhundert nach der Veröffentlichung der »Apriorischen Grundlagen des bürgerlichen Rechts" in etwa in der Lehre vom äußeren und inneren Tatbestand der Willenserklärung wieder ${ }^{81}$. Der große Verdienst Reinachs ist gleichzeitig ein Kritikpunkt: Durch den Anspruch Reinachs, möglichst genau die Beziehungen der sozialen Akte zu den Rechtsgebilden herauszuarbeiten, verfängt er sich, wie bereits dargestellt wurde, teilweise in seinem eigenen System.

Das System Reinachs ist darüber hinaus unvollständig. Reinach benötigt einen erheblichen Begründungsaufwand, um die Selbstgegebenheit der Rechtsgebilde herauszuarbeiten. Er vermag diese Selbstgegebenheit nur durch ein Zusammenwirken

68 REINACH Die apriorischen Grundlagen des bürgerlichen Rechts 722 .

69 Reinach stellt die Vernehmungsbedürftigkeit des Versprechens zur Entstehung einer Verpflichtung im Zusammenhang mit der Entstehung des Anspruchs fest, ohne dies näher zu begründen (REINACH Die apriorischen Grundlagen des bürgerlichen Rechts 722). An anderer Stelle spricht er hingegen davon, dass zwar eine Korrelation von Anspruch und Verbindlichkeit bestünde, eine Verbindlichkeit jedoch auch absolut bestehen kann (REINACH Die apriorischen Grundlagen des bürgerlichen Rechts 698).

70 Reinach spricht auch davon, dass es ein Sich-Verbindlich-Fühlen ohne tatsächliche Verbindlichkeit gebe (REINACH Die apriorischen Grundlagen des bürgerlichen Rechts 696). Dies kann jedoch streng genommen nach den Regeln seines Systems nicht möglich sein. Dem steht auch nicht entgegen, dass Reinach eine Vernehmungsbedürftigkeit der sozialen Akte fordert (REINACH Die apriorischen Grundlagen des bürgerlichen Rechts 708). Der soziale Akt des Versprechens wird ja gerade vom Versprechenden wahrgenommen. Diesen Gedanken hat wohl auch Wilhelm Schapp schon herausgearbeitet, indem er die Verpflichtung zur Übereignung schon nach der diesbezüglichen Äußerung annimmt (WILHELM SCHAPP Der Vertrag als Vorgegebenheit 30).

71 ReINACH Die apriorischen Grundlagen des bürgerlichen Rechts 698. 72 Ebenda.

73 Ebenda 761; auf diese Versprechungen wird nicht mehr eingegangen, da dies den Rahmen der Arbeit sprengen würde.

74 Vgl. hierzu Jan Schapp Sein und Ort der Rechtsgebilde $30 \mathrm{f}$.

75 ReINACH Die apriorischen Grundlagen des bürgerlichen Rechts 693; 734.

76 Reinach Die apriorischen Grundlagen des bürgerlichen Rechts 689.

77 So auch Jan Schapp Sein und Ort der Rechtsgebilde 30, der die apriorischen Sätze allein der Wesenssphäre zuordnet.

78 Reinach Die apriorischen Grundlagen des bürgerlichen Rechts 722, 740.

79 Vgl. hierzu Wilhelm Schapp Der Vertrag als Vorgegebenheit 183; JAN Schapp Sein und Ort der Rechtsgebilde $31 \mathrm{f}$. m. w. N.

80 Reinach Die apriorischen Grundlagen des bürgerlichen Rechts 708.

81 Vgl. hierzu Brox/Walker Allgemeiner Teil des BGB, Rdn. 81; anschaulich bei Schapp/Schur Einführung in das Bürgerliche Recht 4. Aufl. 2006 Rdn. 325. 
von sozialen Akten und Rechtsgebilden zu konstruieren. Eine überzeugende Begründung der Selbstgegebenheit der rechtlichen Gebilde kann dieses System jedoch nicht sein. Der Schwachpunkt liegt darin, dass Reinach die Akte abstrakt als gegeben annimmt, ohne deren Fundierung selbst zu klären ${ }^{82}$. Somit begründet er die Selbstgegebenheit der Rechtsgebilde mit Akten, deren Selbstgegebenheit ihrerseits jedoch noch nicht geklärt ist. Er übersieht somit, dass die Akte selbst einer Fundierung, vergleichbar mit denen der Gegenstände in der Wahrnehmungssphäre, bedürfen ${ }^{83}$.

\section{II. Über die Rechtsphänomenologie von Wilhelm Schapp}

Wilhelm Schapp war ebenso wie Reinach ein Schüler Husserls ${ }^{84}$. Er entwickelte seine Rechtsphänomenologie somit vom gleichen Boden aus, wenn auch mit einer anderen Perspektive ${ }^{85}$. Seine ersten rechtsphänomenologischen Untersuchungen, die »Neue Wissenschaft vom Recht «, I. Band und erst recht der II. Band folgten auf die »apriorischen Grundlagen« Reinachs, sodass er mit der Kritik daran vertraut war und auf sie eingehen konnte ${ }^{86}$. Während »Die neue Wissenschaft vom Recht « in gewisser Weise noch eine Reaktion auf die »Apriorischen Grundlagen « Reinachs darstellt, entfernt sich Wilhelm Schapp in seinen späteren Überlegungen im Rahmen seiner Geschichtenphilosophie von der klassischen Phänomenologie ${ }^{87}$. Jedoch kann man mit Vorsicht sagen, dass sich auch diese Geschichtenphilosophie aus der Sicht Wilhelm Schapps noch innerhalb der Phänomenologie bewegt ${ }^{88}$. Wollte man dieser Auffassung mit gutem Grund nicht folgen ${ }^{89}$, so kann man dennoch davon ausgehen, dass in der Rechtsphänomenologie die spätere Geschichtenphilosophie vorgezeichnet ist $^{90}$. Somit kann auch diese Geschichtenphilosophie bei einer Darstellung der Rechtsphänomenologie Wilhelm Schapps nicht unberücksichtigt bleiben ${ }^{91}$.

\section{Der Vertrag als soziale Gegebenheit}

Wilhelm Schapp beginnt seine Rechtsphänomenologie mit der Untersuchung über den Vertrag ${ }^{92}$. Er unterscheidet zwischen einem vernünftigen und einem vernunftlosen Vertrag sowie der Bestimmung ${ }^{93}$. Der Schwerpunkt der Untersuchung liegt jedoch auf dem gegenseitigen Vertrag ${ }^{94}$. Wilhelm Schapp trennt den Vertrag zunächst in zwei Ebenen. Er unterscheidet zwischen einem Oberbau und einem Unterbau.

\section{a) Die Wertewelt (Unterbau)}

Ausgangspunkt der Überlegungen Wilhelm Schapps ist, dass jeder Vertrag getragen wird "von einem Unterbau von beiderseitigen Überlegungen der Kontrahenten ${ }^{95}$. Diese Überlegungen erfolgen im Wesentlichen in zwei Schritten: Zunächst wird der Wert einer Sache geschätzt ${ }^{96}$, sodann mit dem geschätzten Wert einer anderen Sache verglichen und ein Entschluss gefasst ${ }^{97}$.

\section{aa) Das Schätzen des Wertes einer Sache (Die Wertung)}

Somit kommt im System Wilhelm Schapps zunächst der Wertschätzung eine zentrale Bedeutung zu. Wilhelm Schapp bezeichnet die Wertschätzung als einen ganz eigenartigen »Vorgang zwischen dem Menschen und dem Gegenstand «, der »vielleicht auf der sinnlichen Wahrnehmung und Kenntnis des Gegenstandes" gründet, aber nicht mit der Wahrnehmung selbst zu verwechseln sei $^{98}$. Er macht hierdurch deutlich, dass die Wertschätzung nicht frei von subjektiven Empfindungen sein kann. So kann eine weniger schöne Uhr, die die Sammlung eines Uhrenliebhabers komplettiert, einen höheren Wert für diesen haben als eine neue Dublette $^{99}$. Jedoch ist zu beachten, dass der Wert an sich im Sinne Wilhelm Schapps objektiv ist, im selben Sinne wie ein Gegenstand wahrnehmbar ist ${ }^{100}$. An diesem Punkt bleibt zunächst festzuhalten, dass Wilhelm Schapp seine Überlegungen auf objektiv gegebene Werte stützt. Dies kann nicht deutlich genug hervorgehoben werden, da nach der Auffassung des Verfassers hierdurch ein wesentlicher Schritt vollzogen wurde, der den Überlegungen Reinachs fremd ist. Hierauf wird im weiteren Verlauf noch einzugehen sein.

Somit ist zunächst dieser Wert, dem eine grundlegende Bedeutung zukommt, näher zu untersuchen. Wilhelm Schapp geht davon aus, dass jeder Mensch in seiner eigenen Wertewelt lebt, die er durch das »Genießen « oder das »Kosten« erfassen kann ${ }^{101}$. Dabei übernimmt er bewusst nicht die Methode der Wertermittlung aus der Nationalökonomie, die von einer Konsumtion von Gütern ausgeht ${ }^{102}$. Das »Genießen « sei gewissermaßen ein subjektives Empfinden des Wertes, das »Kosten « eine objektive Herangehensweise zur Ermittlung des Wertes ${ }^{103}$. Wilhelm Schapp macht dies deutlich an einem normalen Menschen, der Wein genießt und einem berufsmäßigen Weinkritiker ${ }^{104}$ : Ein normaler Mensch genießt den Wein und lässt ihn einwirken auf sein subjektives Empfinden, um dadurch den Wert zu bestimmen. Ein

82 Dies wird vor allem auch dadurch deutlich, dass Reinach sogar den Vollzug der Akte von dem Menschen selbst abkoppelt, vgl. JAN SCHAPP Sein und Ort der Rechtsgebilde 39.

83 Im Ergebnis ähnlich auch Wilhelm Schapp Der Vertrag als Vorgegebenheit 182, der jedoch die Kritik nicht explizit auf die Begründung der Selbstgegebenheit bezieht, wohl auch Jan Schapp Sein und Ort der Rechtsgebilde 39, der eine Verankerung des Rechts in der Wertewelt als einen »sehr viel festeren Ausgangspunkt « bezeichnet.

84 Schummann/Smith Adolf Reinach: An intellectual Biography 14.

85 Vgl. hierzu Jan Scha p P Sein und Ort der Rechtsgebilde 38 f; in Bezug auf Jan SCHApp Gödicke Bereicherungsrecht und Dogmatik 182 (Fn. 36).

86 Vgl. nur Wilhelm Schapp Der Vertrag als Vorgegebenheit $182 \mathrm{f}$.

87 Dies wird besonders deutlich bei Wilhelm Schapp In Geschichten verstrickt 169, in dem Wilhelm Schapp selbst seine Überlegungen in Gegensatz zur klassischen Phänomenologie setzt. Auch distanziert er sich mit seiner Geschichtenphilosophie von dem klassischen Phänomenologen Reinach (Wilhelm ScHAPP Philosophie der Geschichten 61).

88 Ebenda, Wilhelm Schapp setzt seine Geschichtenphilosophie nur in den Gegensatz zur klassischen Phänomenologie. Somit scheint er seine eigene Philosophie trotz ihrer grundsätzlichen Unabhängigkeit immer noch der Phänomenologie zuzuordnen.

89 Als Grund könnte zum Beispiel angeführt werden, dass Wilhelm Schapp später die Positionen eines Phänomenologen und die eines Geschichtenphilosophen als unvereinbar gegenüberstellt (Wilhelm Schapp In Geschichten verstrickt 174).

90 GöDICKE Bereicherungsrecht und Dogmatik 182 (Fn. 42).

91 Eine deutliche Unterscheidung der Rechtsphänomenologie und der Geschichtenphilosophie Wilhelm Schapps ist somit nach der hier vertretenen Auffassung nicht angebracht (vgl. GöDICKE Bereicherungsrecht und Dogmatik 182 (Fn. 42), der zwar eine Unterscheidung von Rechtsphänomenologie und Geschichtenphilosophie vornimmt, jedoch zumindest einer Vorzeichnung der Geschichtenphilosophie im Rahmen der Rechtsphänomenologie zustimmt.). Die Arbeit wird sich aufgrund des immensen Umfangs und der klaren Darstellung im Gegensatz zu dem Zusammenwirken von Versprechen, Anspruch und Verbindlich keit im Sinne Reinachs auf die »Neue Wissenschaft vom Recht«, Band I und die entsprechenden Passagen aus den Büchern der Geschichtenphilosophie beschränken.

92 Wilhelm Schapp Der Vertrag als Vorgegebenheit 2.

93 Ebenda.

94 So schon Jan Schapp Sein und Ort der Rechtsgebilde 38. Die Untersuchung wird sich im Folgenden aus den schon in Fn. 82 ausgeführten Gründen auf diesen gegenseitigen Vertrag beschränken. Wenn von nun von »Vertrag« die Rede ist, ist damit der gegenseitige Vertrag gemeint. 95 Wilhelm Schapp Der Vertrag als Vorgegebenheit 6.

96 Ebenda 2, 34

97 Ebenda 34, 56.

98 Ebenda 2.

99 Ebenda 3.

100 Ebenda $8 \mathrm{f}$

101 Ebenda 7.

102 Jan Schapp Sein und Ort der Rechtsgebilde 41 in Bezug auf Wilhelm Schapp Der Vertrag als Vorgegebenheit 11, für die Bestimmung der wertbildenden Faktoren in der Nationalökonomie (heute Volkswirtschaftslehre) vgl. exemplarisch LuCKenBaCH Grundlagen der Volkswirtschaftslehre 1993, $39 \mathrm{ff}$.

103 Wilhelm Schap p Der Vertrag als Vorgegebenheit 8. 104 Ebenda 8. 
berufsmäßiger Kritiker kostet den Wein und vergleicht ihn mit der Qualität anderer Weine und der Nachfrage nach solchen Weinen am Markt. Auch hierdurch ist ein Wert zu ermitteln. Beide Male ist der ermittelte Wert ein objektiver, da er der Sache unmittelbar anhaftet. Da er jedoch durch subjektive Komponenten ermittelt wird, ist er variabel ${ }^{105}$ und daher besonderen Einflüssen ausgesetzt. Insbesondere wird der Wert von der Geschichte bestimmt, in der der Mensch lebt ${ }^{106}$ und der Moral ${ }^{107}$. Dieser Wert, ob gekostet oder genossen, ist Grundlage eines jeden Vertrages.

\section{bb) Das Abwägen von Wertungen (Der Entschluss)}

In einem nächsten Schritt wägt der Vertragspartner den Wert der eigenen Sache gegen den Wert der gegenüberliegenden Sache ab. Dies geschieht im Wesentlichen durch einen Vergleich des Wertes der eigenen mit dem der gegenüberliegenden Sache. Hierfür kann es notwendig sein, dass ein Partner den anderen an seiner Wertewelt teilhaben lassen muss, damit dieser den Wert selbst erfassen kann. Genau dieses Wertverschaffen ist nach Wilhelm Schapp ein Hauptgebiet der Jurisprudenz ${ }^{108}$. Ein weiteres Feld ist der Schutz der Wertewelt gegen Beeinträchtigung, gewissermaßen der Ausgleich bei einem fehlerhaften Aufwiegen durch das Recht der unerlaubten Handlung ${ }^{109}$. Kommt nun dieses Abwägen der gegenseitigen Werte zu dem Ergebnis, dass der Wert der anderen Sache höher ist als der Wert der eigenen, entschließt man sich zum Vertragsschluss ${ }^{110}$. Dieser Vertragsschluss hat daher den Austausch eines Stücks der Wertewelt des einen gegen ein Stück der Wertewelt des anderen zum Inhalt ${ }^{111}$. Die Jurisprudenz beschäftigt sich sodann mit der Frage, wie ein solcher Austausch stattfinden soll ${ }^{112}$.

\section{b) Der Vertragsschluss (Oberbau)}

Diesen Teil des tatsächlichen Vollzugs des Austauschs zwischen den beiden Wertewelten bezeichnet Wilhelm Schapp als Ober$\mathrm{bau}^{113}$. Es sei jedoch schwierig, den genauen Zeitpunkt zu erfassen, in dem nun ein tragfähiger Unterbau vorliege und der Moment erreicht sei, den Vertrag zum Abschluss zu bringen ${ }^{114}$. Er denkt sich hierzu die Kontrahenten in einen rechtsleeren Raum und lässt sie einen Austauschvertrag vollziehen. Dazu seien zumindest Kundgebungen von beiden Parteien, eine Verpflichtung einzugehen, notwendig ${ }^{115}$. Dies ist auch der Weg, den das BGB gewählt hat: Gem. $\$ 151$ S. 1 HS. 1 kommt ein Vertrag durch die Annahme des Antrags zustande. Somit bleibt festzuhalten, dass im Oberbau zwei Willenserklärungen zum Vollzug des Wertaustauschs notwendig sind ${ }^{116}$. Somit ist es eine vorrechtliche Gegebenheit des Vertrages, dass er nur durch die Kundgabe zweier Willenserklärungen entstehen kann ${ }^{117}$.

Das Zusammenwirken von Oberbau und Unterbau wird deutlich bei der Frage, welche Art von Vertrag zustande kommt. Es hängt allein von der kundgegebenen Wertschätzung beider Parteien ab, ob beispielsweise ein Kauf oder eine Schenkung vollzogen wird ${ }^{118}$. Schätzen beispielsweise beide Parteien der Sache den gleichen Wert bei und geben trotzdem kund, dem anderen den Teil seiner Wertewelt übertragen zu wollen, so liegt eine Schenkung vor ${ }^{119}$.

Wilhelm Schapp betrachtet nun diese Willenserklärungen näher. Er kommt zu dem Schluss, dass diese Willenserklärungen soziale Akte seien ${ }^{120}$. Wilhelm Schapp definiert jedoch im Gegensatz zu Reinach sehr wohl, was er unter "sozialen Akten " versteht: Soziale Akte seien "psychische und interpsychische Vorgänge, d. h. Vorgänge, die sich zugleich in der Seele und zwischen den Seelen abspielen. . $^{121}$ Er arbeitet nun nicht wie Reinach diese Akte in all ihrer Verschiedenheit heraus, sondern nimmt sie gewissermaßen als gegeben an. Dies legt den Schluss nahe, dass sich Wilhelm Schapp bei dem Vollzug des Vertragsschlusses im Oberbau ganz Reinach angeschlossen hat ${ }^{122}$. Insoweit kann für eine weitergehende Darstellung des Vertragsschlusses des Ober- baus durch den Vollzug von sozialen Akten grundsätzlich auf die Lehren Reinachs verwiesen werden. Wilhelm Schapp geht wie Reinach davon aus, dass das positive Recht grundsätzlich nicht durch die Vorgegebenheiten vorgezeichnet ist. Es findet vielmehr diese Vorgegebenheiten vor und wählt aus ihnen aus ${ }^{123}$. Somit sei ein Vertrag beispielsweise ein natürliches Gebilde, dessen sich die Gesetzgebung lediglich bemächtigt habe ${ }^{124}$. Dennoch ist zu erwähnen, dass Wilhelm Schapp einen anderen Anknüpfungspunkt der sozialen Akte als Reinach wählt. Wilhelm Schapp verankert die sozialen Akte, beispielhaft das Versprechen, in der sozialen Wirklichkeit ${ }^{125}$. Somit sucht Wilhelm Schapp nicht wie Reinach die Selbstgegebenheit der sozialen Akte durch ein seltsames System zu belegen, sondern er knüpft bewusst an die soziale Wirklichkeit an ${ }^{126}$.

\section{Zwischenergebnis}

Der Vertragsschluss findet bei Wilhelm Schapp somit insgesamt in drei Schritten statt ${ }^{127}$ : Zunächst wird eine Sache gewertet. "Auf der Grundlage dieser Wertungen werden Entschlüsse gefasst. Die Entschlüsse führen zu einem Stadium, in welchem die Abgabe von Willenserklärungen in Frage kommt.« Diese Willenserklärungen münden sodann in einen Vertrag. Die Ebene der Wertungen wird als »Unterbau« bezeichnet, die Ebene des Vertragsschlusses als »Oberbau«. Man könnte diese zwei Ebenen mit den Wörtern »Ob« und »Wie« kennzeichnen. Der Unterbau enthält alle Charakteristika, die für das »Ob« des Vertragsschlusses vonnöten sind. Der Oberbau hingegen beschäftigt sich mit dem »Wie«. Das »Wie« ist von Reinach eingehend erörtert worden. Wilhelm Schapp beschäftigt sich eingehend mit dem »Ob«.

\section{Der Vertrag als Geschichte}

Wie eben ausgeführt wurde, knüpft das System Wilhelm Schapps an die vorangegangenen Untersuchungen Reinachs an. Dennoch

105 Sonst würde ein Abschätzen auch keinen Sinn machen.

106 Wilhelm Schapp Der Vertrag als Vorgegebenheit 16; Ders. In Geschichten verstrickt $1 \mathrm{ff}$.; Wilhelm Schapp macht das unter anderem deutlich am Beispiel der Wertewelt Homers, die sich zu Zeiten Platos schon verlagert habe und spätestens durch die Stoiker gänzlich verbannt wurde (Wilhelm Schapp Der Vertrag als Vorgegebenheit 17).

107 Wilhelm Schapp Der Vertrag als Vorgegebenheit 14

108 Ebenda 22.

109 Ebenda, vgl. auch GöDICKE Bereicherungsrecht und Dogmatik 184.

110 Wilhelm Schapp Der Vertrag als Vorgegebenheit 2 ff.; vgl. auch JAN SCHAPP Sein und Ort der Rechtsgebilde 38, der hier jedoch von Leistungen statt von Werten spricht und GöDICKE Bereicherungsrecht und Dogmatik 182.

111 Wilhelm Schapp Der Vertrag als Vorgegebenheit 27.

112 Ebenda.

113 Ebenda 34: »Bei allen [Verträgen, Ergänzung durch Autor] besteht der Unterbau in der Abschätzung der umzusetzenden Werte und der Oberbau in Kundgebungen, welche diese Umsetzungen bewirken."

114 Ebenda 29.

115 Ebenda 30.

116 Ebenda 56.

117 Diese Herangehensweise ist bezeichnend für die Methode Wilhelm Schapps: Durch den Blick auf die soziale Wirklichkeit reduziert er den Vertrag (und später auch Eigentum und unerlaubte Handlung) auf die wesentlichen, vorrechtlichen Gegebenheiten. (GöDICKE Bereicherungsrecht und Dogmatik 184).

118 Wilhelm Schapp Der Vertrag als Vorgegebenheit 31.

119 Ebenda 31.

120 Ebenda 56.

121 Ebenda.

122 Jan Schapp Sein und Ort der Rechtsgebilde 38

123 Wilhelm Schapp Der Vertrag als Vorgegebenheit 5, $35 \mathrm{f}$

124 Ebenda 5.

125 Ebenda 56.

126 Vgl. auch die Gegenüberstellung bei Jan Schapp Sein und Ort der Rechtsgebilde 39, zustimmend GöDICKe Bereicherungsrecht und Dogmatik 182 (Fn. 36).

127 Vgl. hierzu Wilhelm Schapp Der Vertrag als Vorgegebenheit 56. 
ist erkennbar, dass in der im Gegensatz zu Reinach gänzlich anderen Darstellung der Rechtsgebilde, fußend auf einer in der sozialen Wirklichkeit tatsächlich ablaufenden chronologischen Abfolge eines Vertragsschlusses, bereits neue Gedanken Wilhelm Schapps vorgezeichnet sind ${ }^{128}$. Den Abschluss finden diese Gedanken dann in der Geschichtenphilosophie Wilhelm Schapps, in der er den Vertrag vom Beginn der Verhandlungen bis zum Abschluss als Geschichte auffasst ${ }^{129}$. Aus dieser Sicht ist erst recht keine mühselige Konstruktion einer Selbstgegebenheit der sozialen Akte oder der Rechtsgebilde nötig, wie sie Reinach vorgenommen hat. Aus der Sicht Wilhelm Schapps ist "das Verstricktsein in Geschichten der letzte Ort [...] für die Rede von Wirklichkeit ${ }^{130}$ Somit ist die Geschichte in diesem Sinne absolut.

Diese Überlegungen machen jedoch nur dann Sinn, wenn man sie aus der Sicht eines klassischen Rechtsphänomenologen wie Reinach anstellt. In dem Werk »Der Vertrag als Vorgegebenheit « hatte Wilhelm Schapp eben gerade diesen Ausgangspunkt ${ }^{131}$, in seiner Geschichtenphilosophie sucht er jedoch nicht mehr die Nähe Reinachs und distanziert sich sogar von ihm ${ }^{132}$. Somit wechselt Wilhelm Schapp die Perspektive. Zentraler Anknüpfungspunkt sind nicht mehr wie in der klassischen Phänomenologie die Gegenstände und die Wahrnehmung derjenigen, sondern der in die Geschichte Verstrickte ${ }^{133}$. Somit kommt dem Vertragsschließenden entscheidende Bedeutung in der Geschichtenphilosophie zu. Hier wählt Wilhelm Schapp nun als Anknüpfungspunkt gänzlich die soziale Wirklichkeit ${ }^{134}$. Der Vertrag sei somit eben nicht in Gegebenheiten begründet, sondern er wird durch seine Geschichte und durch die darin Verstrickten ausgeformt ${ }^{135}$. Hier eröffnet Wilhelm Schapp nun ein neues Feld, dessen Darstellung über den Rahmen dieser Arbeit hinausgehen würde ${ }^{136}$.

\section{Die Rechtsphänomenologie von Wilhelm Schapp im Vergleich zur apriorischer Rechtslehre von Reinach}

Die Affinität der klassischen Rechtsphänomenologie Wilhelm Schapps zu Reinachs »Apriorischen Grundlagen des bürgerlichen Rechts « wird insbesondere durch die weitgehende Übernahme des Rechtslehre Reinachs in den Oberbau bei Wilhelm Schapp deutlich ${ }^{137}$. Wilhelm Schapp nähert sich jedoch dieser Erkenntnis aus einer anderen Richtung als Reinach. Letzterer versucht, aus der »Wirklichkeit in eine Wesenssphäre zu gelangen«, während Wilhelm Schapp von vornherein die Verankerung der Gebilde in der sozialen Wirklichkeit sucht ${ }^{138}$.

Durch die Erkenntnis, dass jedem Vertragsschluss als Oberbau eine Wertung und ein Entschluss als Unterbau vorausgeht, erscheinen der Vertragsschluss und damit auch die sozialen Akte in einem neuen Licht. Während Reinach die Selbstgegebenheit der rechtlichen Gebilde nur durch ein kompliziertes System aus sozialen Akten herzuleiten versucht, baut Wilhelm Schapp mit der Erkenntnis des Unterbaus gewissermaßen das Fundament für die Lehre Reinachs. Wilhelm Schapp erkennt die Fundierung der sozialen Akte in den Wertungsgrundlagen ${ }^{139}$. Dieser Wert an sich ist objektiv und durch seine Genießbarkeit auch in gewissem Sinne wahrnehmbar, sodass er als selbstgegeben im phänomenologischen Sinne gelten kann. Durch diese "Verankerung « der Rechtsgebilde »in der sozialen Wirklichkeit« hat Wilhelm Schapp »einen sehr viel festeren Ausgangspunkt als Reinach $\ll^{140}$.

In der Geschichtenphilosophie distanziert sich Wilhelm Schapp dann ausdrücklich von Reinach ${ }^{141}$ und nimmt eine gänzlich neue Perspektive ein. Der Vertrag sei eben gerade nicht in Gegebenheiten begründet ${ }^{142}$. Ausgangspunkt sei vielmehr die Vertragsgeschichte selbst und der darin Verstrickte.

\section{Schluss und Kritik}

Somit wirft das anfangs thematisierte Dasein des Rechts erwartungsgemäß mehr Fragen auf, als beantwortet werden können. Einerseits kann man Recht als Vorgegebenheit verstehen und sich hierbei beispielsweise der aufgezeigten Begründungsansätze bedienen. Andererseits könnte man Recht auch als Geschichte verstehen, die maßgeblich durch die darin Verstrickten gestaltet wird.

Sowohl Reinach als auch Schapp verkennen bei ihrer Analyse, dass sie ein Ergebnis präsentieren, dass sie selbst als Präsentierenden nicht einschließt. Die Auswirkung ihrer eigenen Rolle ist nicht von den jeweiligen Theorien erfasst. So kommt es dazu, dass beide Theorien einen »blinden Fleck $^{143}$ aufweisen, der eine Subjektivierung der Erkenntnisse nach eigenen Werten nicht ausschließt. Sowohl Schapp als auch Reinach waren anfangs von dem »erkenntnisleitenden Interesse ${ }^{144}$ erfasst, eine Selbstgegebenheit der von ihnen dargestellten Rechtsgebilde zu erarbeiten. Sie gehen dabei nicht darauf ein, dass sie möglicherweise ihre Theorien erst aufstellen konnten, weil sie bereits durch die Gesellschaft und ihr Studium schon in der Weise vorgeprägt und dadurch in der Lage waren, bestimmte Sachverhalte gedanklich beispielsweise als »Vertrag« zu gruppieren. Beide reflektieren nicht die Auswirkungen ihrer eigenen Rolle als Beobachter. Aus diesem Grund bleiben ihre Theorien unvollständig, es bleibt bei dem Vorhandensein eines »blinden Flecks«. Ein solcher »blinder Fleck« entsteht jedoch bei allen Theoriebildungen, die auf Beobachtung fußen ${ }^{145}$. Er wird verursacht durch die Tatsache, dass der Beobachter sich nicht selbst beobachten kann, wie es ein Dritter könnte ${ }^{146}$. Daher bleiben die von Reinach und Schapp präsentierten Lösungen das, was sie am wenigsten sein möchten: Konstruktionen, die sich aufgrund ihrer systemimmanenten Unzulänglichkeit durch die Rolle des Beobachters nur näherungsweise an die Realität als »Horizont « herantasten können ${ }^{147}$. Dennoch bleibt festzuhalten, dass diese Realität existiert. Mithin ist nicht ausgeschlossen, dass Schapp und Reinach tatsächlich Recht behalten und solche Rechtsgebilde existieren. Wir werden es selbst jedoch nie wirklich wissen können. Wir können uns eben nur dabei beobachten, wie wir Realität konstruieren $^{148}$.

128 So im Ergebnis auch GöDICKE Bereicherungsrecht und Dogmatik 182 (Fn. 42).

129 Wilhelm Schapp Philosophie der Geschichten 2. Aufl. 1981, 60.

130 Wilhelm Schapp In Geschichten Verstrickt 182.

131 Wilhelm Schapp Der Vertrag als Vorgegebenheit 182, hier sucht er einen Anknüpfungspunkt zu Reinach.

132 Wilhelm Schap Philosophie der Geschichten 61

133 Wilhelm Schapp In Geschichten Verstrickt 1.

134 Wilhelm Schapp Philosophie der Geschichten 62.

135 Ebenda.

136 Für einen Überblick vgl. Jan Schapp Sein und Ort der Rechtsgebilde $117 \mathrm{ff}$.

137 Jan SCHApp Sein und Ort der Rechtsgebilde 38.

138 Ebenda 39, zustimmend GöDICKE Bereichungsrecht und Dogmatik 182 (Fn. 36).

139 Wilhelm Schapp Der Vertrag als Vorgegebenheit 182.

140 Jan Schapp Sein und Ort der Rechtsgebilde 39.

141 Wilhelm Schapp Philosophie der Geschichten 61

142 Ebenda 62.

143 Vgl. auch Baecker (Hrsg.) Luhmann Einführung in die Systemtheorie 2002, 146.

144 Ha веRmas Erkenntnis und Interesse, Frankfurt am Main 1968.

145 Berghaus Luhmann leicht gemacht 48 weist darauf hin, dass »die Gesetzmäßigkeit, dass jede Beobachtung einen systemrelevanten blinden Fleck erzeugt « nicht zu durchbrechen sei.

146 Vgl. auch Baecker (Hrsg.) LuhmanN Einführung in die Systemtheorie 2002, 146.

147 Luhmann Die Realität der Massenmedien 2. Aufl. 1996, 18 f.

148 Ebenda. 